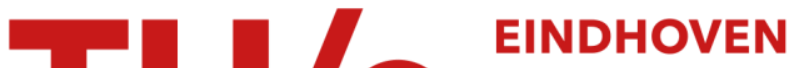 UNIVERSITY OF TECHNOLOGY
}

\section{Inkjet Nozzle Failure by Heterogeneous Nucleation}

\section{Citation for published version (APA):}

Fraters, A., Van Den Berg, M., De Loore, Y., Reinten, H., Wijshoff, H., Lohse, D., Versluis, M., \& Segers, T. (2019). Inkjet Nozzle Failure by Heterogeneous Nucleation: Bubble Entrainment, Cavitation, and Diffusive Growth. Physical Review Applied, 12(6), [064019]. https://doi.org/10.1103/PhysRevApplied.12.064019

DOI:

10.1103/PhysRevApplied.12.064019

Document status and date:

Published: 09/12/2019

\section{Document Version:}

Publisher's PDF, also known as Version of Record (includes final page, issue and volume numbers)

\section{Please check the document version of this publication:}

- A submitted manuscript is the version of the article upon submission and before peer-review. There can be important differences between the submitted version and the official published version of record. People interested in the research are advised to contact the author for the final version of the publication, or visit the $\mathrm{DOI}$ to the publisher's website.

- The final author version and the galley proof are versions of the publication after peer review.

- The final published version features the final layout of the paper including the volume, issue and page numbers.

Link to publication

\section{General rights}

Copyright and moral rights for the publications made accessible in the public portal are retained by the authors and/or other copyright owners and it is a condition of accessing publications that users recognise and abide by the legal requirements associated with these rights.

- Users may download and print one copy of any publication from the public portal for the purpose of private study or research.

- You may not further distribute the material or use it for any profit-making activity or commercial gain

- You may freely distribute the URL identifying the publication in the public portal.

If the publication is distributed under the terms of Article 25fa of the Dutch Copyright Act, indicated by the "Taverne" license above, please follow below link for the End User Agreement:

www.tue.nl/taverne

Take down policy

If you believe that this document breaches copyright please contact us at:

openaccess@tue.nl

providing details and we will investigate your claim. 


\title{
Inkjet Nozzle Failure by Heterogeneous Nucleation: Bubble Entrainment, Cavitation, and Diffusive Growth
}

\author{
Arjan Fraters $\odot,{ }^{1}$ Marc van den Berg, ${ }^{2}$ Youri de Loore, ${ }^{2}$ Hans Reinten, ${ }^{2}$ Herman Wijshoff, $, 2,3$ \\ Detlef Lohse, ${ }^{1}$ Michel Versluis, ${ }^{1}$ and Tim Segers ${ }^{1, *}$ \\ ${ }^{1}$ Physics of Fluids Group, Max-Planck Center Twente for Complex Fluid Dynamics, Department of Science and \\ Technology, MESA+ Institute, and J. M. Burgers Centre for Fluid Dynamics, University of Twente, P.O. Box 217, \\ Enschede 7500 AE, Netherlands \\ ${ }^{2}$ Océ Technologies B.V., P.O. Box 101, Venlo 5900 MA, Netherlands \\ ${ }^{3}$ Department of Mechanical Engineering, Eindhoven University of Technology, P.O. Box 513, Eindhoven 5600 \\ $M B$, Netherlands
}

(Received 30 June 2019; revised manuscript received 17 October 2019; published 9 December 2019)

\begin{abstract}
Piezoacoustic drop-on-demand (DOD) inkjet printing is widely applied in high-end digital printing due to its unprecedented precision and reproducibility. Micron-sized droplets of a wide range of chemical compositions can be deposited; however, the stability of piezoacoustic DOD inkjet printing can sometimes be compromised through the stochastic entrainment of bubbles within the ink channel. Here, bubble nucleation, translation, and growth are studied in an experimental silicon-based printhead with a glass nozzle plate using high-speed imaging that is triggered by changes in the ink-channel acoustics. It is found that impurities in the ink can trigger bubble nucleation upon their interaction with the oscillating meniscus. Cavitation inception on a dirt particle during the rarefaction pressure wave is identified as a second mechanism for bubble formation. The acoustic driving pressure within the ink channel, and its change upon bubble nucleation, are obtained from a fit of a Rayleigh-Plesset-type bubble-dynamics equation to the measured time-resolved radial dynamics of the bubble. The measured decrease in channel resonance frequency after bubble entrainment results in a $24 \%$ increased ink-jet length. The nucleated bubbles translate toward the ink-channel walls due to acoustic radiation forces and ink streaming. The convective ink flow is characterized using high-speed particle-tracking velocimetry. The vortical flow near the oscillating meniscus is shown to trap the impurities, thereby increasing the particle-to-meniscus interaction probability and, correspondingly, the bubble-entrainment probability.
\end{abstract}

DOI: 10.1103/PhysRevApplied.12.064019

\section{INTRODUCTION}

Inkjet printing is a successful industrial application of microfluidics [1-3]. It allows for highly controlled noncontact material deposition at picoliter volumes. The high versatility of piezo-driven drop-on-demand (DOD) inkjet printing results from the wide range of inks that can be jetted. Consequently, it is widely used in an industrial setting for document printing, for printing graphic arts, and for package printing [1]. More recently, inkjet technology has successfully been employed for additive manufacturing, including electronic components [4-12], pharmaceuticals [13], biomaterials [14-17], and ceramic components [18].

Earlier piezo-driven printheads were fabricated through micromachining. These ink channels had a typical length of the order of centimeters [1]. The pressure buildup at the nozzle entrance required to jet a droplet has

\footnotetext{
*t.j.segers@utwente.nl
}

been generated through positive interference of traveling acoustic waves [1,19]. More recently, the inkjet industry has developed printheads using microelectromechanicalsystems (MEMS) technology [20-23]. MEMS technology allows for lower fabrication costs with respect to micromachining techniques, a higher fabrication precision, a higher nozzle density, and more efficient and well-controlled jetting at high DOD frequencies. MEMS-based ink channels have dimensions much smaller than the wavelength of the acoustic driving pulse. The resulting channel acoustics is that of a Helmholtz resonator [24], i.e., it is comparable to a classical mass-spring system. The resonance frequency is governed by the mass of ink in the nozzle and restrictor and by the compliance of the ink chamber, i.e., that of the ink and that of the wall including the membrane with the thin-film piezoactuator $[1,2]$.

The stability and reproducibility of droplet formation is crucial for reliable inkjet-printing applications. Typically, billions of droplets can be jetted at a very high 
reproducibility. However, it has been shown that the jet reproducibility is sometimes compromised by the entrainment of air bubbles in the ink channel [21,22,25-30]. An entrained bubble disturbs the ink-channel acoustics and thereby droplet formation. At a later stage, when the bubble has grown through rectified diffusion [31-34], its compressibility can even counteract the pressure buildup in the ink channel such that droplet formation stops. Once a bubble is entrained, it can practically only be eliminated through diffusive dissolution, i.e., by switching off the piezoactuation for a period of the order of seconds to minutes $[22,26]$.

To increase the stability of the inkjet printing process, it is crucial to understand the physical mechanisms by which bubbles are formed and entrained in the ink channel. Potential formation mechanisms include cavitation inception in the rarefaction pressure wave [33], bubble entrainment by direct contact of dirt particles in the ink channel with the meniscus, and bubble pinch-off from a meniscus instability, e.g., a Rayleigh-Taylor instability [35,36], a parametrically driven instability [37], or a directional instability of the ink jet by defects in the nozzle [38]. For a classic micromachined printhead developed at Océ Technologies B.V. it has been shown that dirt particles, transported by a flow of ink on the wetted nozzle plate and moving layers of ink on the nozzle plate can induce bubble entrainment upon their interaction with a jetting nozzle [25]. However, it has also been observed by the authors that the probability of bubble entrainment remains nonzero even for printheads with an antiwetting nozzle plate that prevents transport of dirt particles and ink to jetting nozzles. Thus, other physical mechanisms for bubble entrainment have yet to be revealed.

In order to understand the bubble-entrainment mechanism, it is key to visualize the bubble-entrainment process on the microseconds time scale. The stochastic nature of the bubble-entrainment process requires precise triggering to such an entrainment event. It has been shown that the piezoactuator can be used as a pressure sensor and that changes of the ink-channel acoustics can be used to trigger a high-speed optical recording [25]. Visualization is challenging, as these printheads are fabricated through micromachining or fabricated in silicon and they are opaque to visible light. Silicon is partly transparent to infrared (IR) light, allowing for infrared visualization of the inside of a MEMS-based ink channel [22,39]. Nevertheless, the high refractive index of silicon $(n=3.42)$ complicates IR imaging of the nozzle region due to total (internal) reflection at the silicon interfaces [22]. Furthermore, to date, the maximum temporal resolution achieved by IR cameras is only of the order of $1 \mathrm{~ms}$, whereas the jetting process, and the whole bubble-entrainment process, takes place on a microseconds time scale. Thus, to date, IR imaging has limited use for the study of bubble entrainment in MEMS-based printheads in real time.
Optical access to visible light has previously been obtained for a classic micromachined printhead by the intrusive addition of glass-etched channels with glassetched nozzles to the printhead [26]. In this system, bubbles could be visualized in the ink channel. However, the temporal resolution of the imaging system at that time was only $25 \mu$ s, i.e., 1 order of magnitude too low to image the bubble-entrainment process itself. Another limitation at that time was that the powder-blasting method led to hourglass-shaped ink channels with different acoustic and flow properties as compared to the standard ink channels.

In this study, an etching technique is applied to produce a printhead with an optically transparent glass nozzle plate. Stochastic bubble entrainment in a jetting ink channel is imaged simultaneously with the jetting process by using two high-speed cameras triggered on changes in the ink-channel acoustics. Entrained bubbles are used as a pressure sensor and their translation, as well as their growth and effect on the jetting process, are studied. Furthermore, particle-tracking-velocimetry experiments are used to characterize the convective ink flow inside the ink channel. The obtained flow field is used to explain the trajectories of dirt particles near the nozzle entrance. Finally, bubble entrainment is imaged at high driving amplitudes and the failure statistics are measured in a first attempt to reveal the physical mechanism through which disturbing bubbles are entrained.

\section{EXPERIMENTAL METHODS}

\section{A. Printhead and ink}

The experimental printhead has a silicon-based functional acoustic part connected to an optically transparent $500-\mu \mathrm{m}$-thick fused-silica nozzle-plate chip (see Fig. 1). The channel features in the fused-silica chip are fabricated
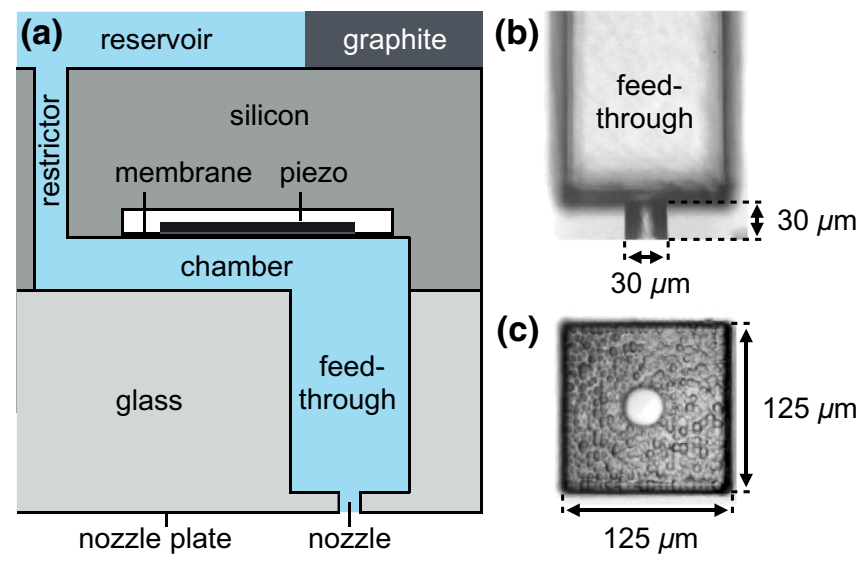

FIG. 1. (a) A schematic side view of the ink channel in the silicon printhead with a glass nozzle plate. (b) Side-view and (c) bottom-view microscope images of the empty ink channel in the glass nozzle plate. 


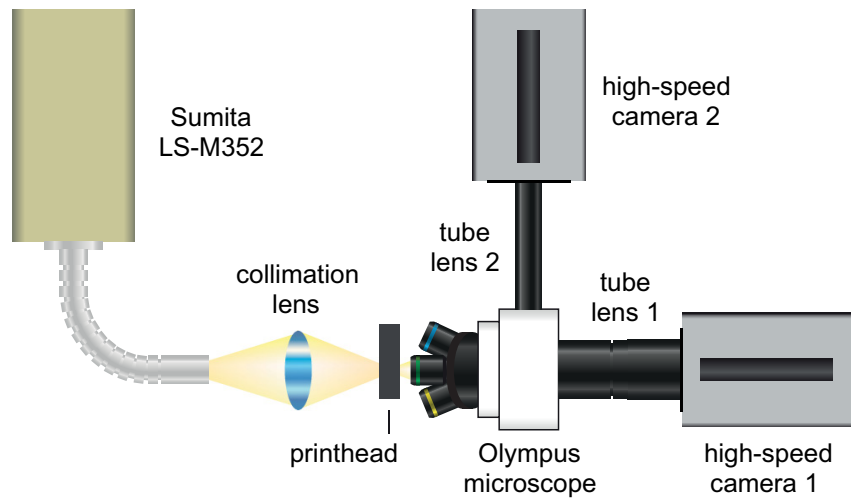

FIG. 2. The high-speed imaging setup employed to simultaneously visualize droplet formation and bubble entrainment.

at a precision of $1 \mu \mathrm{m}$ through femtosecond-laser-pulse printing and subsequent chemical etching (FemtoPrint, Muzzano, Switzerland). Figures 1(b) and 1(c) show side and bottom micrographs of the channels in the fused-silica chip. The nozzles are cylindrical, with a diameter and length of $30 \mu \mathrm{m}$. Further details regarding the printhead fabrication are provided in Appendix A.

\section{B. Imaging setup}

The printhead is imaged using a modular microscope (BXFM-F, BXFM-ILHS, Olympus) equipped with a $20 \times($ SLMPLN20x, Olympus) and a 50× (SLMPLN50x, Olympus) magnifying objective, as shown in Fig. 2. Due to the optical-path-length difference between the inside and the outside of the printhead, two high-speed cameras with slightly different optical-focal-plane positions are used to image the inside of the ink channel (camera 1) and the droplet formation outside of the printhead (camera 2), respectively. A 50:50 beam splitter is used to display the microscope image on both high-speed camera 1 (HPV-X2, Shimadzu or SA-X2, Photron) and high-speed camera 2 (SA1.1, Photron). Illumination is provided by a continuous fiber-optic light source (Sumita LS-M352). For details regarding the temporal resolution, see Appendix B. During the experiments, the cameras are continuously recording using their ring-buffer memory. Once a trigger is received, the cameras stop recording and the frames before and after the trigger pulse in their respective memories are used to reveal the bubble-entrainment event.

\section{Camera-triggering system}

A triggering system is developed that generates a trigger pulse once a bubble is entrained. Similarly to what has previously been reported [25], the trigger pulse is generated when a change in the channel acoustics is detected. A field-programmable gate array (FPGA) compares the measured acoustic signals of the channel in real time to a reference signal that is obtained from a normal jetting nozzle before each experiment. A trigger pulse is generated once the correlation coefficient is smaller than 0.9. Full details regarding the implementation of the triggering system are provided in Appendix C.

\section{Image analysis}

The high-speed videos obtained from the triggered cameras are processed using an automated image-analysis algorithm programmed in MATLAB. The radial dynamics of the bubble are obtained from the inflection point of its intensity profile [40]. For each frame, the center position of the bubble (based on the center of mass) is stored to track its displacement over time. The jet velocity is measured from the frontal position of the head of the droplet, which is tracked over time, and its velocity is calculated at a $500 \mu \mathrm{m}$ distance from the nozzle exit.

\section{E. Acoustic driving pressure}

The radial dynamics of an acoustically driven bubble is described by a Rayleigh-Plesset type equation $[32,41]$ :

$$
\begin{aligned}
\rho\left(\ddot{R} R+\frac{3}{2} \dot{R}^{2}\right)= & \left(P_{0}+\frac{2 \sigma}{R_{0}}\right)\left(\frac{R_{0}}{R}\right)^{3 \kappa}\left(1-\frac{3 \kappa \dot{R}}{c}\right) \\
& -P_{0}-P(t)-4 \mu \frac{\dot{R}}{R}-\frac{2 \sigma}{R} \\
& -\rho \frac{\partial}{\partial t}\left(\frac{\dot{R} R^{2}}{2 d}\right),
\end{aligned}
$$

where $\rho$ is the ink density, $R(t)$ is the bubble radius, $P_{0}$ is the ambient pressure in the ink channel, $\sigma$ is the interfacial surface tension of the air-ink interface, $R_{0}$ is the equilibrium bubble radius, $\kappa$ is the polytropic gas constant, $c$ is the speed of sound in the ink, $P(t)$ is the acoustic pressure, and $\mu$ is the dynamic ink viscosity. The time derivatives are denoted by the overdots. The final term in Eq. (1) accounts for the vicinity of a rigid wall, with $d$ being the distance from the bubble center to the wall [41], to approximate the pressure contribution of the reflection at the ink-channel wall. Here, $d$ is taken as the distance between the center of the bubble and the nearest wall during the measurement, i.e., the bottom of the feedthrough. Distance $d$ changes within one actuation cycle and its instantaneous value is used.

Equation (1) can be linearized to obtain the resonance frequency $f_{R}$ of a bubble at a wall, i.e., for $d=R$, as follows [32,41]:

$$
f_{R}=\frac{\sqrt{2 / 3}}{2 \pi} \sqrt{\frac{1}{\rho R_{0}^{2}}\left(3 \kappa P_{0}+(3 \kappa-1) \frac{2 \sigma}{R_{0}}\right)}=\sqrt{\frac{2}{3}} f_{0},
$$

where $f_{0}$ is the resonance frequency of the bubble in the unbounded fluid. Thus, the resonance frequency of the bubble at a rigid wall decreases by a factor of $\sqrt{\frac{2}{3}} \approx 0.82$. 
The solution of Eq. (1), $R(t)$, can be fitted to the measured radial dynamics of a bubble inside an ink channel to obtain the acoustic driving pressure $P(t)$. The trapezoidal piezoactuation pulse drives the ink channel into resonance such that the acoustic driving-pressure pulse in the ink channel may be approximated by a sinusoid multiplied by a Gaussian, as follows:

$$
P(t)=P_{A} \sin (\omega t) \exp \left[-\frac{1}{2}\left(\frac{t-t^{*}}{\Omega}\right)^{2}\right],
$$

where $P_{A}$ is the pressure amplitude, $\omega=2 \pi f$ the angular frequency, and $t$ is time. The variance of the Gaussian $\Omega$ and the time at which it is at a maximum, $t^{*}$, are fitting parameters. The FMINSEARCH function in MATLAB is used to find the driving-pressure pulse that results in a best fit of Eq. (1) to the measured radial dynamics of the bubble.

\section{F. Probability distribution of dirt-particle position}

Experiments are also performed in an ink channel without bubbles, to study the behavior of the dirt particles present in the ink. The probability distribution of the dirtparticle positions in the ink channel is obtained from a 10-min optical recording (SA-X2, 1 frame $/ \mathrm{s}, 0.4 \mu \mathrm{m} /$ pixel, $2.5 \mu$ s exposure time) of an ink channel jetting droplets at a DOD frequency of $20 \mathrm{kHz}$ and a velocity of $8 \mathrm{~m} / \mathrm{s}$. The recorded images are processed as described in Appendix D.

\section{G. Particle-tracking velocimetry}

To explain the observed dirt-particle trajectories and the probability distribution of the dirt-particle position, the flow of the ink in the channel is studied through particletracking-velocimetry (PTV) measurements by adding 0.01 vol $\%$ of $1-\mu$ m-diameter $\mathrm{SiO}_{2}$ particles to the ink. The ink channel filled with the particle-laden ink is imaged at a frame rate of 50000 frames/s ( $5 \mu \mathrm{s}$ shutter time, HPV-X2 camera) at a spatial resolution $0.6 \mu \mathrm{m} /$ pixel. The velocity field is measured from the recordings in an automated way, as detailed in Appendix E.

\section{H. Bubble nucleation at a high driving amplitude}

The imaging setup with the continuous light source is not able to capture all of the important details of the bubble-nucleation process because the maximum frame rate at which bubble nucleation can be recorded at sufficiently high image quality, $1 \times 10^{6} \mathrm{frames} / \mathrm{s}$, is limited by the brightness of the continuous light source. Therefore, additional bubble-nucleation events are recorded at a higher frame rate of $5 \times 10^{6}$ frames/s (HPV-X2 camera), at a spatial resolution of $0.6 \mu \mathrm{m} /$ pixel and using a brighter pulsed-xenon light source. However, as a result of the limited maximum repetition rate of the xenon-flash light source $(10 \mathrm{~Hz})$, the printhead has to be operated far outside the regular operating range for nozzle failure to occur within a reasonable amount of time. The flashes are fired exactly at the start of the first meniscus retraction during every actuation cycle, thereby limiting the DOD frequency to $10 \mathrm{~Hz}$, which is 2000 times lower than the typical DOD frequency. The employed jet velocity is fixed at almost $20 \mathrm{~m} / \mathrm{s}$, more than twice the typical regular jet velocity, such that on average only 9000 droplets have to be jetted before nozzle failure, corresponding to $15 \mathrm{~min}$.

\section{Nozzle-failure statistics}

In an attempt to reveal the nature of the bubblenucleation mechanism, the statistics of nozzle failure is measured as a function of the jet velocity. The failure statistics are typically plotted as a function of the jet velocity to aid comparison of the failure statistics measured for different printheads with a potentially different piezo sensitivity. Therefore, first, the jet velocity is characterized as a function of the piezo driving voltage using highspeed imaging. Then, the number of successfully jetted droplets before nozzle failure is measured as a function of the piezo driving voltage, using high-speed recordings at 200 frames/s (SA-X2 camera, spatial resolution $0.4 \mu \mathrm{m} / \mathrm{pixel})$. At each driving voltage, the failure experiment is repeated ten times. A detailed description of the failure-statistics measurement is given in Appendix F.

\section{RESULTS}

\section{A. Air entrainment by bubble nucleation on a dirt particle}

Figures 3(a) and 3(c) show typical bubble-entrainment events in an ink channel jetting at a DOD frequency of $20 \mathrm{kHz}(10 \mathrm{~m} / \mathrm{s}$ jet velocity), imaged at a temporal resolution of $2 \mu \mathrm{s}$ using the continuous light source and a shutter time of $1 \mu \mathrm{s}$. The timings of the piezoactuation pulse, the positions of the meniscus, and the entrainment events are shown in Fig. 3(b). Note that in both cases a dirt particle is present that translates toward the oscillating meniscus. Once the particle is located close to the meniscus, a bubble nucleates on the dirt particle. Note that for both nucleation events, the nucleation bubble becomes visible at the start of the second meniscus retraction of the actuation cycle. During the next actuation cycle, the bubble detaches from the particle and translates toward a wall of the ink channel. In total, more than 50 bubble-nucleation events are imaged and in all cases at least one dirt particle is present close to the meniscus during bubble nucleation (for more information on the dirt particles, see Sec. E). Furthermore, in $83 \%$ of all imaged nucleation events, the bubble appears at the start of the second meniscus retraction. In the remaining cases, the bubble appears at the start of the first meniscus retraction of an actuation cycle. 


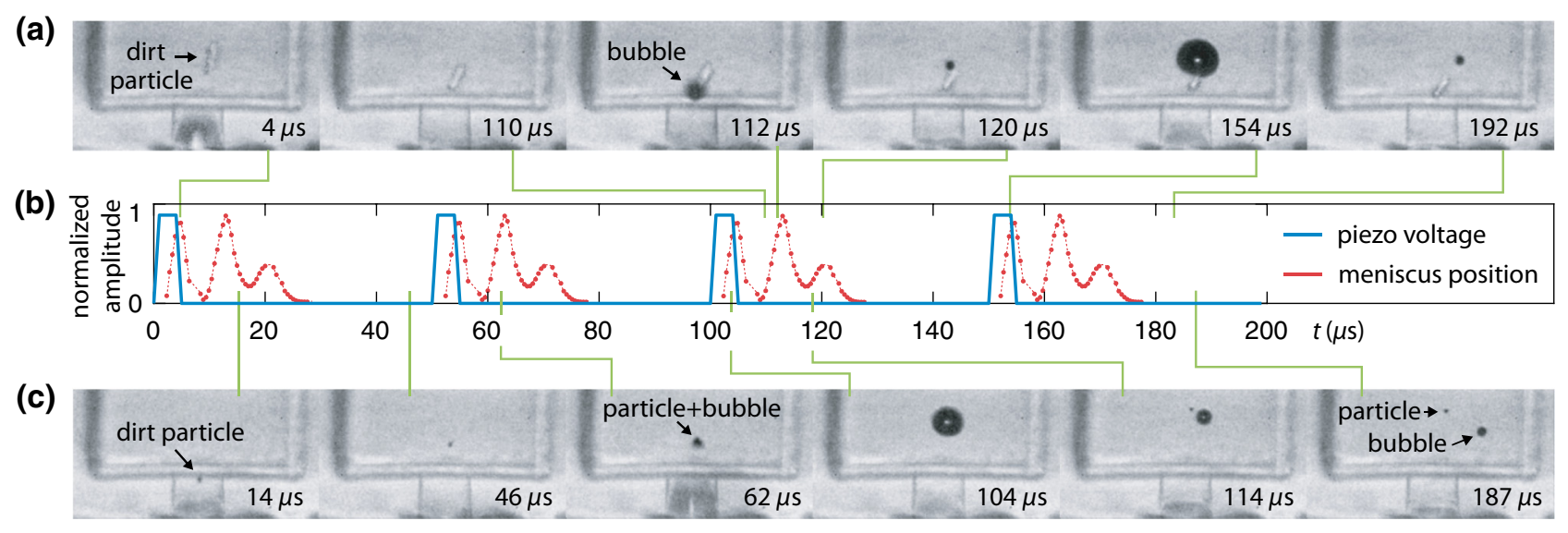

FIG. 3. (a),(c) Typical bubble-nucleation events. A dirt particle translates toward the jetting meniscus and a bubble nucleates on the particle. After the next actuation cycle, the bubble and particle separate. See also Videos 1 and 2 in the Supplemental Material [42]. (b) The timing of the piezoactuation pulse and the position of the meniscus. The timing at which the images in (a) and (c) are taken with respect to the actuation pulse and corresponding meniscus motion is indicated by the solid green lines.

\section{B. The nucleated bubble as a pressure sensor}

The radial dynamics of a nucleated bubble is measured at a temporal resolution of $1 \mu \mathrm{s}[0.5 \mu$ s shutter time; see Fig. 4(a)] and is used to derive the driving-pressure pulse inside the ink channel. The measured radial dynamics

(a)
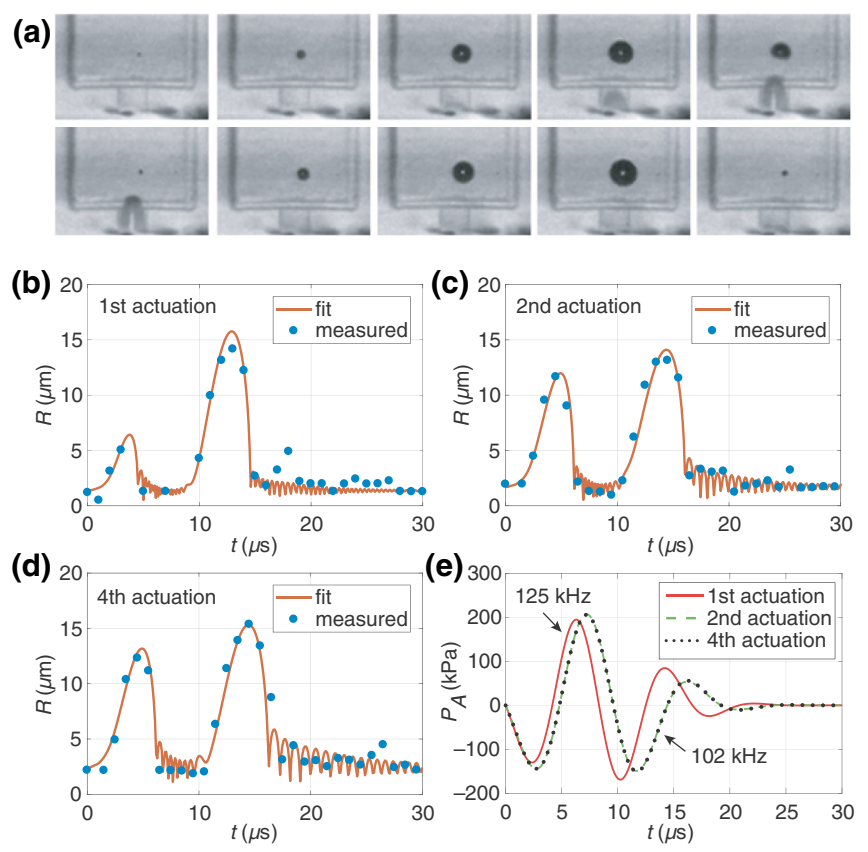

FIG. 4. (a) The radial dynamics of a nucleated bubble recorded at a temporal resolution of $1 \mu \mathrm{s}$. See also Video 3 in the Supplemental Material [42]. (b) The measured and fitted radial dynamics for a nucleated bubble during (b) the first actuation, (c) the second actuation, and (d) the fourth actuation after bubble appearance. (e) The acoustic driving pressure as a function of time, calculated using the Rayleigh-Plesset equation based on the fitted radial dynamics of the bubble. and the fitted radial dynamics are plotted in Fig. 4(b) for the first piezoactuation pulse where the bubble is visible. Figures 4(c) and 4(d) show the measured and fitted radialbubble dynamics for the second and fourth piezoactuation pulses after the bubble appears. Note that the equilibrium bubble size $R_{0}$ increases over the four piezo actuations from $1.3 \mu \mathrm{m}$, to $1.7 \mu \mathrm{m}$, to $2.1 \mu \mathrm{m}$, to $2.2 \mu \mathrm{m}$, respectively, a result of rectified diffusion [31-34]. Also note that, in all cases, the second bubble expansion is of larger amplitude than the first one, indicating that the rarefaction pressure is larger during the second meniscus retraction. Indeed, the obtained driving-pressure pulses [Fig. 4(e)] show that the rarefaction pressure during the first meniscus retraction is $125 \mathrm{kPa}$ and that during the second meniscus retraction it is $150 \mathrm{kPa}$. The higher rarefaction pressure during the second meniscus retraction may explain the higher bubble-nucleation probability that is reported in the previous section. Furthermore, note that the frequency of the acoustic driving-pressure pulses decreases by $18 \%$, namely from $125 \mathrm{kHz}$ to $102 \mathrm{kHz}$, after the first actuation pulse. Thus the nucleated bubble decreases the eigenfrequency of the ink channel. The frequency and amplitude of the driving-pressure pulse do not change during the two subsequent actuation pulses.

Figures 4(b)-4(d) show that the bubble oscillations in the ink channel are highly nonlinear, with bubble excursions more than 7 times larger than the equilibrium bubble size, resulting in an inertial bubble collapse [34]. Note from the modeled curves that after the collapse, the bubble ring-down frequency is much higher than that of the driving ultrasound pulse. Indeed, using Eq. (2), it can be calculated that the resonance frequency of a microbubble with a radius of the order of $2 \mu \mathrm{m}$ is $1.4-1.8 \mathrm{MHz}$, depending on the stand-off distance to the wall, i.e., 1 order of magnitude higher than the frequency of the ink-channel 


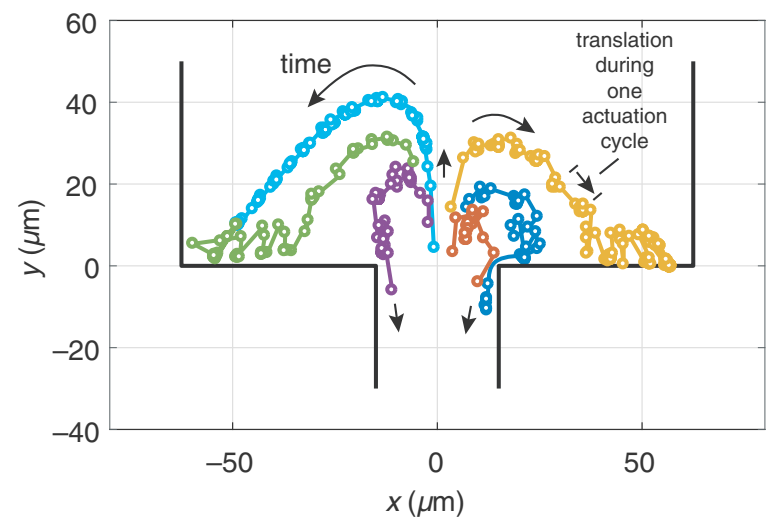

FIG. 5. The translational movement of the nucleated bubbles. Some bubbles are jetted outward (purple, red, and blue curves) and others translate into the ink channel (light blue, green, and yellow curves).

acoustics. In this calculation, a polytropic exponent of 1.4 is used, assuming air-filled bubbles and adiabatic bubble oscillations. Thus, as expected, the resonance frequency of the bubble is much higher than that of the Helmholtzresonance frequency of the ink channel.

\section{Bubble translation}

Figure 5 shows the translation of the nucleated bubble for six different bubble-nucleation events, imaged at a temporal resolution of $2 \mu \mathrm{s}$ and with a shutter time of $1 \mu \mathrm{s}$. The translation direction is indicated by the arrows. Note that the cross section of the feedthrough is square and that the translational motion of the bubble may in part be out of the imaging plane. The nucleated bubbles first translate into the ink channel (the positive $y$ direction). During the subsequent actuation cycles, the bubble can be jetted outward (the purple, red, and blue curves) or it can be entrained into the ink channel through its translation toward a channel wall (the light blue, green, and yellow curves). The distance between the groups of data points in Fig. 5 indicates the distance over which the nucleation bubbles translate during one actuation cycle. For the measurements given in Fig. 5 the bubbles arrive at the channel wall within 15 actuation cycles, i.e., within $750 \mu$ s. The translational paths of the bubbles are discussed in Sec. IV.

\section{Jet formation during bubble nucleation and growth}

In this section, the role of bubble nucleation and bubble growth on inkjet formation is investigated. Two highspeed cameras are used to image both the jet formation outside the printhead and the bubble-nucleation events inside the ink channel. Figure 6(a) shows an image sequence of the inkjet before, during, and after the nucleation of a bubble. Figure 6(b) shows the inside of the ink channel with a dirt particle on which a nucleated bubble is present
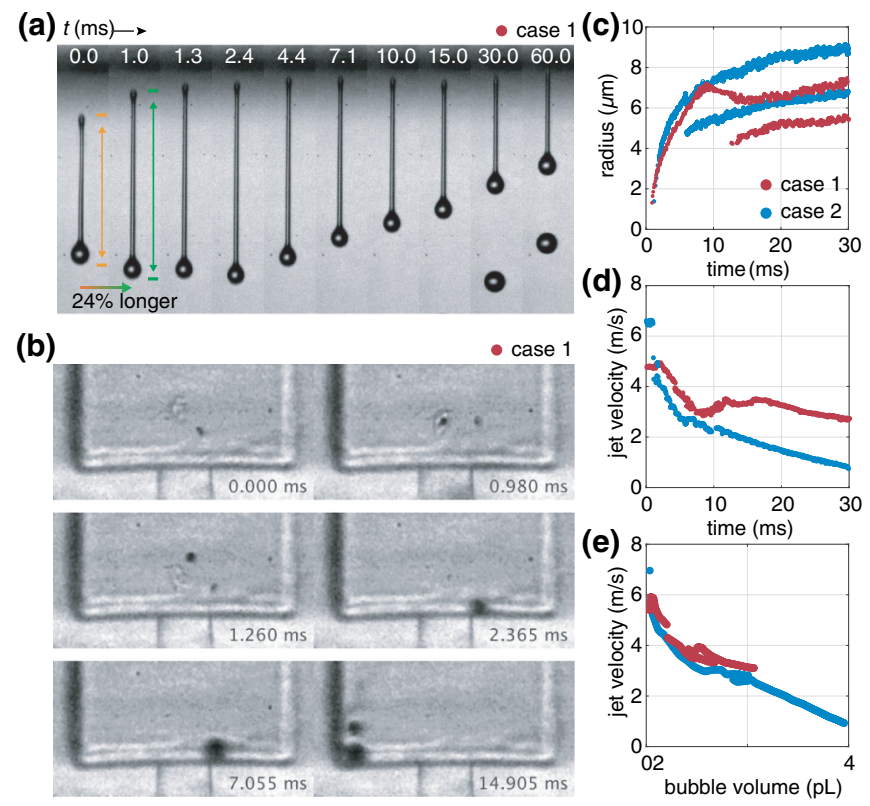

FIG. 6. (a) The image sequence of a jetting nozzle during the nucleation and growth of a bubble. (b) The bubble nucleation and growth are imaged simultaneously with the jetting nozzle. The bubble nucleates at $t=0.98 \mathrm{~ms}$. (c) The bubble radius as a function of time for two nucleation events. In both cases, the nucleated bubble splits into two bubbles after several milliseconds, probably due to fragmentation as a result of jet formation in the bubble during strong asymmetric collapse toward the wall [33]. (d) The jet velocity as a function of time. (e) The total bubble volume versus the jet-velocity curves of both cases seem to collapse onto the same curve. See also Video 4 in the Supplemental Material [42].

$(t=0.98 \mathrm{~ms})$. The first droplet that is formed after bubble nucleation $(t=1.0 \mathrm{~ms})$ is $24 \%$ longer than the droplet formed under normal conditions $(t=0.0 \mathrm{~ms})$.

After nucleation, the bubble translates toward the channel wall, where it arrives at $t=2.3 \mathrm{~ms}$. The bubble grows over time [the red dots in Fig. 6(c)] and at $t=12 \mathrm{~ms}$ it breaks up into two bubbles that both translate toward the left corner of the channel, where they arrive at $t=14.9 \mathrm{~ms}$. To show that the nucleation and growth of a bubble is a nonuniversal process, a second bubble-entrainment case is also plotted in Fig. 6(c). Qualitatively, cases 1 and 2 correspond well, i.e., a bubble nucleates, grows, and breaks up into two bubbles. However, the growth rate and break-up time are different.

The growing bubbles decrease the jet velocity, as can be seen in Fig. 6(d), where the jet velocity is plotted as a function of time for both nucleation cases. Despite the measured differences in bubble growth and jet velocity, Fig. 6(e) shows that for both cases the total bubble-volume versus jet-velocity curves seem to be correlated; however, more experimental data are required for verification. 

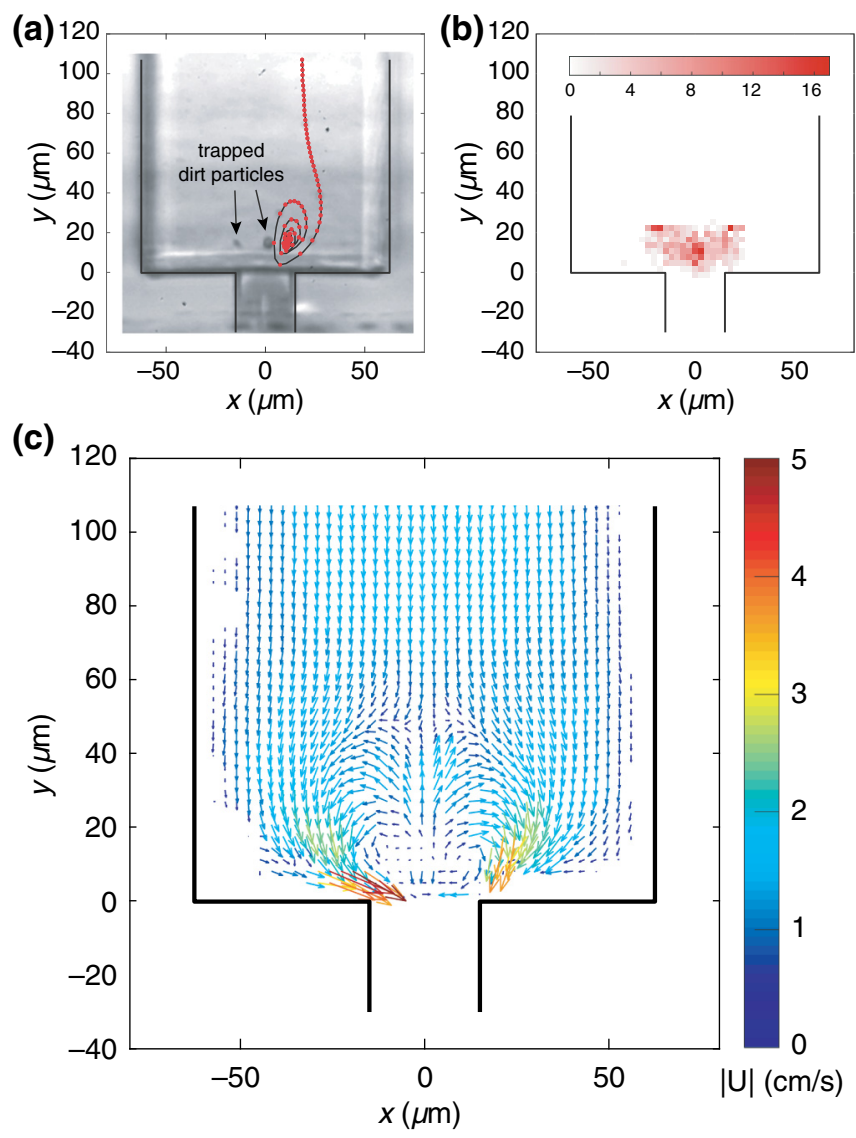

FIG. 7. (a) The path of a dirt particle that gets trapped in a region in front of the nozzle, recorded at a frame rate of 10000 frames/s and with a shutter time of $5 \mu \mathrm{s}$. (b) The probability distribution of the dirt-particle position, based on 680 dirt-particle positions. The color indicates the number of particles found in the grid cell. (c) The time-averaged velocity field inside the ink channel measured by PTV, showing a vortex ring in front of the nozzle. See also Video 5 in the Supplemental Material [42].

\section{E. Dirt-particle trapping and ink velocity field}

In this subsection, we show that dirt particles that are present in the ink channel may get trapped in the region near the nozzle, inside the ink channel. A typical example of the translation of a dirt particle that is trapped is plotted in Fig. 7(a). Note that the dirt particle originates from the ink upstream of the feedthrough. Once the dirt particle approaches the nozzle, it enters a spiral directed inward to a fixed point near the nozzle. Also note in Fig. 7(a) that there are two more dirt particles present at the same distance from the nozzle entrance (indicated by the arrows).

In total, the locations of 680 dirt particles are measured from images of a jetting nozzle. Figure 7(b) shows the probability of the dirt-particle positions inside the ink channel. The color indicates the number of particles found in a grid cell. Clearly, dirt particles are trapped in the region near the nozzle with a projected area (height $\times$ width) of approximately $25 \times 50 \mu \mathrm{m}^{2}$.
To explain the entrapment of dirt particles near the nozzle, the time-averaged flow velocity field in an ink channel without bubbles is measured using particle-tracking velocimetry (PTV). The results are presented in Fig. 7(c). Near the oscillating meniscus, a vortex ring is observed, i.e., at the axisymmetry axis $(x=0)$ of the ink channel, the flow is directed upward in the positive $y$ direction and it recirculates off axis. Acoustic streaming near an oscillating interface is a well-known phenomenon [43] and it results in a vortex ring [44-46]. Note, by comparison of Figs. 7(a) and 7(c), that the dirt particles are trapped in the center of the vortex ring where the recirculation velocity is at a minimum.

To estimate the density of the dirt particles and the physical trapping mechanism, the terminal sedimentation velocity of approximately spherical dirt particles is measured at a frame rate of 60 frames/s in a nonactuated ink channel. The particles considered have diameters ranging from $5 \mu \mathrm{m}$ to $12 \mu \mathrm{m}$. By equating the Stokes drag to the gravitational force, the particle density is calculated to be approximately $2500 \mathrm{~kg} / \mathrm{m}^{3}$, i.e., 2.3 times higher than the density of the surrounding ink.

\section{F. Bubble nucleation by cavitation}

In a first attempt to reveal the mechanism by which a bubble nucleates on a dirt particle, the nucleation event during a high-amplitude driving pulse is imaged at $5 \times 10^{6}$ frames/s using a xenon single-flash light source. Figure 8 shows a typical image sequence of a bubble-nucleation event. Note that the ink contains seeding particles and note the dirt particle near the nozzle entrance in the first frame at $t=0 \mu \mathrm{s}$. During the first meniscus retraction, the dirt particle remains located at the channel wall and it does not come into direct contact with the meniscus. When the meniscus moves outward, the inkjet is formed and the flow of ink drags the dirt particle along into the nozzle (see $t=6.2 \mu \mathrm{s})$. When the particle moves past the sharp corner into the nozzle, a bubble cavitates hydrodynamically on the particle due the low local pressure resulting from the high local ink velocity. Thus, it is shown here that, at this extremely high driving amplitude, dirt particles do not have to be in direct contact with the ink-air meniscus to nucleate a bubble.

\section{G. Nozzle-failure statistics}

To further characterize the bubble-nucleation mechanism, the nucleation probability is studied as a function of the driving amplitude of the ink channel. Before the nucleation probability is studied, the dependence of the jet velocity and the meniscus amplitude are studied as a function of the piezo driving amplitude. First, Fig. 9(a) shows that the jet velocity is approximately linearly dependent on the driving amplitude of the piezo. Figure 9(b) shows the maximum meniscus retraction, or inward amplitude, with 


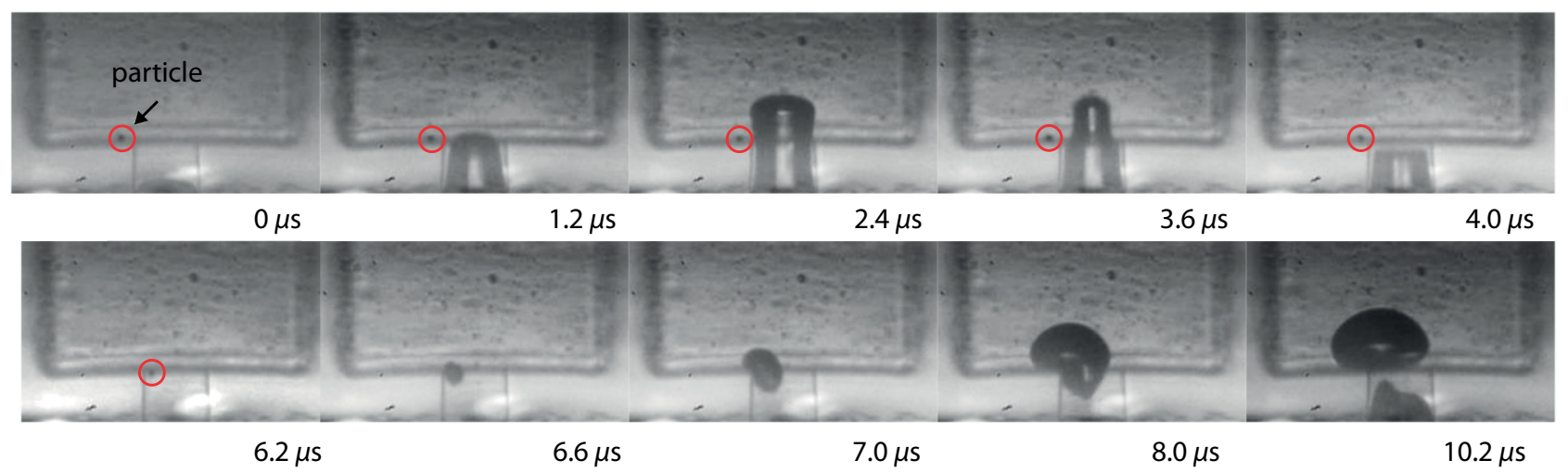

FIG. 8. Cavitation on a particle. The meniscus retracts for the first time and subsequently ejects a droplet. The ejection of an ink jet results in the acceleration of the ink. The particle, initially located at the wall, is dragged along by the ink, first toward the nozzle and then around the edge into the nozzle. The increased ink velocity and resulting low local pressure result in hydrodynamic cavitation on the dirt particle starting at $t=6.6 \mu \mathrm{s}$. See also Video 6 in the Supplemental Material [42].

respect to the nozzle exit. The figure shows that the inward amplitude of the meniscus is also approximately linearly dependent on the driving voltage of the piezo.

The nozzle-failure probability measured as a function of the jet velocity is shown in the box plot in Fig. 9(c). The stability of the inkjet printing process is measured at seven different jet velocities by measuring at each jet velocity, for 10 times, the number of droplets that can be successfully jetted before nozzle failure. In Fig. 9(c), for each data set per jet velocity, the lowest measured probability is represented by the lower horizontal bar, the first quartile by the lower value of the box, the median by the bar inside the box, the third quartile by the upper limit of the box, and the maximum measured value by the upper horizontal bar. Note that the nozzle-failure probability increases approximately exponentially with the jet velocity over the measured jet-velocity range.

\section{DISCUSSION}

In this work, it is shown that bubbles nucleate on dirt particles (Fig. 3). The affinity of the gas with the dirt particles and the observed cavitation on the particles suggest that the dirt particles are inkophobic or, at least, only partially wetting. As a result, nucleation sites are always present on the particles, i.e., formed through the diffusion of dissolved gas toward the dirt particles. Moreover, the measured density of $2500 \mathrm{~kg} / \mathrm{m}^{3}$ suggests that the dirt particles are composed of silicon or glass, likely originating from the unconventional printhead assembly.

Due to the density mismatch of the dirt particles with the surrounding ink, the dirt particles may penetrate the meniscus interface to directly collect air across the free interface. To investigate this, the Stokes number is calculated, as follows [47]: $S t k=t_{0} u_{0} / l_{0}$, where $t_{0}=\rho_{p} d_{p}^{2} / 18 \mu$ is the relaxation time of the particle, $u_{0}$ is the ink velocity, and $l_{0}$ is the particle diameter $(6-8 \mu \mathrm{m})$. The maximum ink velocity is assumed to be twice the jet velocity $(5-10 \mathrm{~m} / \mathrm{s})$. The resulting Stokes number is 1.0-2.0. Thus, indeed, a dirt particle with a Stokes number $>1$ located close to the nozzle may get into direct contact with the meniscus, since it does not closely follow the streamlines during fast advancement and retraction of the meniscus.

Apart from bubble nucleation through direct interaction of dirt particles with the meniscus, it is shown in Fig. 8 that bubble nucleation is also possible through cavitation in the bulk liquid, without direct contact with the meniscus. The increased ink velocity in the nozzle decreases the pressure according to Bernoulli's law [48] and the pressure drop triggers the nucleation. However, it must be noted that these cavitation experiments are performed at twice the normal driving amplitude and, therefore, the nucleation mechanism may be different for a printhead operated under normal conditions. Nevertheless, future work may explore the jet stability of inkjet nozzles with rounded corners in order to lower the hydrodynamic pressure drop to minimize the occurrence of edge cavitation.

The measured failure probability is shown to exponentially increase with the jet velocity [Fig. 9(c)] and this cannot be explained on the basis of the linear relationships between the jet velocity and the driving voltage and between the meniscus retraction and the driving voltage [Figs. 9(a) and 9(b)]. The exponential increase hints toward a Gaussian-distributed probability of bubble nucleation versus the jet velocity, since the cumulative probability-distribution function corresponding to a Gaussian increases exponentially with the jet velocity in the low-probability range of Fig. 9(c). However, the two identified entrainment mechanisms may both be at play. Therefore, for future model development, the actual entrainment mechanism needs to be characterized for different jet velocities under improved illumination conditions. Only then can we explain the exponential increase of the failure-probability curve. 
(a)

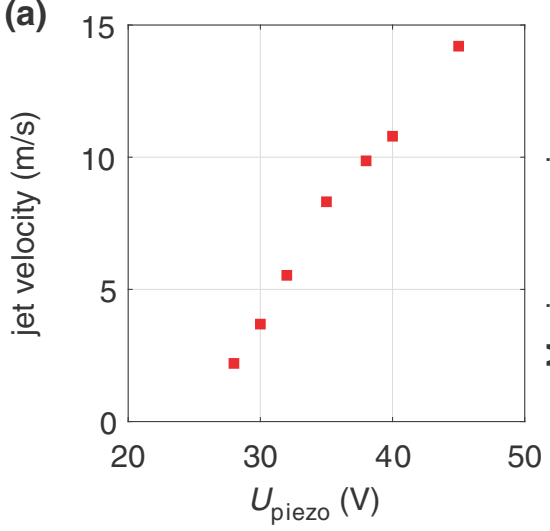

(b)

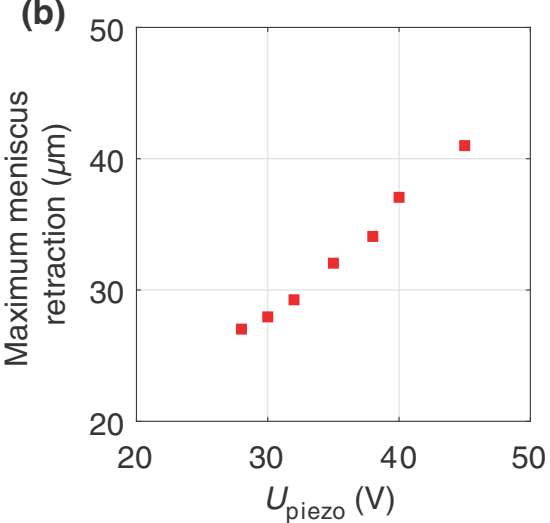

(c)

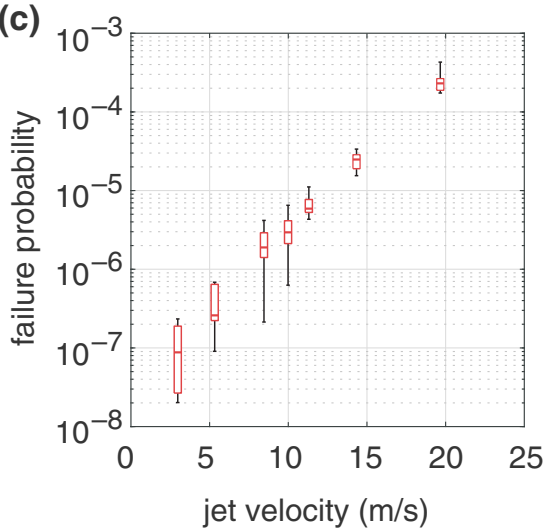

FIG. 9. (a) The jet velocity and (b) the maximum meniscus retraction as function of the piezo driving-pulse amplitude. (c) The measured failure statistics as a function of the jet velocity. For each data set per jet velocity, the lowest measured probability is represented by the lower horizontal bar, the first quartile by the lower value of the box, the median by the bar inside the box, the third quartile by the upper limit of the box, and the maximum measured value by the upper horizontal bar. The high dirt-particle concentration results in a high failure probability as compared to standard printheads produced using cleanroom facilities.

In this work, dirt particles are shown to be trapped in the vortex near the nozzle. This trapping is surprising, since the centrifugal force on the particles with a density higher than that of the ink is directed away from the center of the vortex [49]. An explanation for this discrepancy is that the shear-gradient lift force and/or the rotational lift force [4951] are the dominating trapping forces. Future work should focus on understanding the trapping forces - e.g., by using numerical simulations - and on the mechanisms by which the vortex can be momentarily disturbed, e.g., by using a different piezo driving waveform, in order to jet dirt particles outward, thereby increasing the jet stability.

The translational paths of the nucleated bubbles can be qualitatively explained by a combination of acoustic radiation forces and hydrodynamic forces $[32,52,53]$. The freshly nucleated bubble is pushed into the ink channel by two mechanisms. First, the primary Bjerknes force pushes the nonresonant bubble into the ink channel, toward the pressure antinode at the piezo, where the acoustic pressure amplitude is higher than that at the nozzle entrance. Second, the convective ink flow [Fig. 7(c)] drags the nucleation bubble along and upward into the ink channel. Then, the secondary radiation force becomes dominant on the bubble that has now grown due to rectified diffusion, such that it is attracted toward the acoustically reflective channel walls [54]. Estimation of the relative contributions of the forces is challenging since the bubble dynamics are highly nonlinear, with large amplitudes of oscillation. Therefore, the relative contributions of the acoustic and hydrodynamic forces should be computed through numerical modeling in future work.

The length of the jet formed after bubble nucleation is observed to increase by $24 \%$, whereas the jet velocity increases by only $3 \%$ (Fig. 6). The increased jet length can be explained on the basis of the decrease in the frequency of the acoustic driving-pressure wave inside the ink channel. The acoustic driving frequency is measured to decrease by $18 \%$ after bubble nucleation. The decreased driving frequency and the corresponding meniscus motion result in an extension of the time window over which ink is ejected out of the ink channel by $18 \%$; in other words, the droplet formation process takes $18 \%$ longer. Together with the 3\% higher jet velocity, which also results in a longer ink jet, a $22 \%$ longer jet is expected, in good agreement with the measured $24 \%$ increase in jet length. At longer time scales, the jet velocity decreases due to the compressibility of the bigger gas bubble, which prevents pressure buildup at the nozzle [26].

\section{CONCLUSIONS}

Stochastic air-bubble entrainment and droplet formation are visualized simultaneously in an optically transparent piezoacoustic inkjet printhead through high-speed imaging. The high-speed cameras are triggered by changes in the ink-channel acoustics due to bubble entrainment. It is found that dirt particles trigger bubble nucleation upon their interaction with the oscillating meniscus. Direct contact of a particle with the meniscus during fast advancement and retraction of the meniscus and cavitation on a particle during a rarefaction pressure wave are identified as the key mechanisms through which a bubble is formed. Furthermore, an exponential relation is found between the jet velocity and the nozzle-failure probability. Upon nucleation, the bubble translates into the ink channel, separates from the dirt particle, and either moves to a corner of the ink channel or returns to the nozzle, where it is jetted outward. The fate of the bubble depends on where it nucleates within the vortical flow field. 
The dirt particles originate from inside the ink channel and are trapped in a vortex flow in front of the nozzle, thereby increasing the particle-meniscus interaction probability. This vortex flow is revealed by characterizing the ink-velocity field using particle-tracking velocimetry. The driving-pressure pulse in the ink channel is characterized from a fit of the measured radial dynamics of the bubble to the Rayleigh-Plesset bubble-dynamics equation. The nucleated bubble causes the ink jet to be temporarily $24 \%$ longer, mainly due to a corresponding decrease in the ink-channel resonance frequency.

\section{ACKNOWLEDGMENTS}

We thank the employees from Océ Technologies B.V. and Philips Innovation Services who assisted in the production of the printhead; in particular, Henk Simons, Norbert Lamers, Jos Lemmen, Henk Stolk, and Maikel Huygens from Océ Technologies B.V. and Theo Michielsen from Philips Innovation Services. This work is part of the research program "High Tech Systems and Materials" (HTSM) with Project No. 12802, and is part of the Industrial Partnership Program No. 143 of the Dutch Technology Foundation (STW) and the Foundation for Fundamental Research on Matter (FOM), which are part of the Netherlands Organisation for Scientific Research (NWO). The research was cofinanced by Océ Technologies B.V., the University of Twente, and Eindhoven University of Technology.

\section{APPENDIX A: PRINTHEAD}

The experimental printhead is produced with the help of Océ Technologies B.V. and Philips Innovation Services. Due to the unconventional character of the printhead configuration, it is assembled outside the cleanroom. This results in impurities that pollute the ink channels, due to which these channels have a higher bubble-entrainment probability than regular printheads. An antiwetting coating consisting of self-assembled monolayers of (tridecafluoro-1,1,2,2tetrahydrooctyl)trichlorosilane (FOTS, abcr GmbH, CAS 78560-45-9) is applied to the outside of the fused-silica chip through which the imaging is performed, such that the formation of an optically disturbing ink layer is prevented. The employed ink is hot-melt ink (nonpigmented CrystalPointŮ, Océ Technologies B.V.), which is jetted from the nozzles at a temperature of $130^{\circ} \mathrm{C}$. At this temperature, the ink viscosity, density, and surface tension are $10 \mathrm{mPa} \cdot \mathrm{s}, 1080 \mathrm{~kg} / \mathrm{m}^{3}$, and $28 \mathrm{mN} / \mathrm{m}$, respectively.

\section{APPENDIX B: IMAGING SETUP}

The temporal resolution at which the bubble entrainment can be visualized using the employed setup with the HPV-X2 camera and the continuous light source is $1 \mu \mathrm{s}$ at a spatial resolution of $1.5 \mu \mathrm{m} /$ pixel $(20 \times$ magnification) and $2 \mu \mathrm{s}$ at a spatial resolution of $0.6 \mu \mathrm{m} / \operatorname{pixel}(50 \times$ magnification). The temporal resolution is lower at the higher spatial resolution because of the limited back-light illumination intensity.

For the simultaneous recordings of the bubble entrainment and the droplet formation, SA-X2 and SA1.1 cameras are used, both of which have a spatial resolution of $1.0 \mu \mathrm{m} /$ pixel $(20 \times$ magnification). The bubble entrainment is imaged by the SA-X2 camera at a temporal resolution of $5 \mu \mathrm{s}(1 \mu \mathrm{s}$ shutter time $)$ and the droplet formation is imaged by the SA1.1 camera at a temporal resolution of $12.5 \mu \mathrm{s}$ (1 $\mu \mathrm{s}$ shutter time).

\section{APPENDIX C: PIEZO ACTUATION AND CAMERA TRIGGERING SYSTEM}

The field-programmable gate array (FPGA, National Instruments, NI PXI-7952R) is programmed such that it generates a Pearson correlation coefficient [55] for each piezo-actuation pulse. Figure 10 shows a schematic representation of the triggering and piezo-actuation system. A pulse-delay generator (Berkeley Nucleonics Corp., BNC 575) triggers an arbitrary-waveform generator (AWB, Wavetek 195) at the $20-\mathrm{kHz}$ DOD frequency. Furthermore, the pulse-delay generator sends a clock signal with a frequency of $10 \mathrm{MHz}$ to the waveform generator to synchronize the two devices to avoid jitter on the FPGA sampling signal. Upon receipt of each trigger, the AWG produces three waveforms. The first waveform is the standby signal for the FPGA. The second waveform is a $2-\mathrm{MHz}$ FPGA sampling signal consisting of 64 pulses corresponding to the 64 data points that the FPGA records per acoustic sensing signal. The third waveform is the trapezoidal piezo driving waveform. The piezo of the ink channel is driven at a DOD frequency of $20 \mathrm{kHz}$ by a trapezoidal waveform with a rise and fall time of $1 \mu \mathrm{s}$ and a high time of $3.0 \mu \mathrm{s}$, optimized for the ink-channel Helmholtz resonance frequency of $125 \mathrm{kHz}$. The piezo driving pulse is amplified to $38 \mathrm{~V}$ (corresponding to a $10 \mathrm{~m} / \mathrm{s}$ droplet velocity) by a wideband power amplifier (Krohn-Hite 7602M) during the bubble-entrainment experiments and is connected to the printhead via a custom-made electronic switch. The switch connects the piezo driving pulse to the piezoactuator and switches toward the analog-to-digital converter (ADC, National Instruments, NI 5732) once the piezo signal drops below $1 \mathrm{~V}$. The digital signal is recorded by the FPGA and compared in real time to a reference signal that is obtained from a normal jetting nozzle before each experiment. A trigger pulse is generated once the correlation coefficient is smaller than 0.9. The FPGA firmware is programmed in the graphical programming language LABVIEW (National Instruments) and compiled using a Xilinx compiler into a bit file, after which it is loaded to the FPGA. Moreover, during the experiments, LABVIEW 
(a)

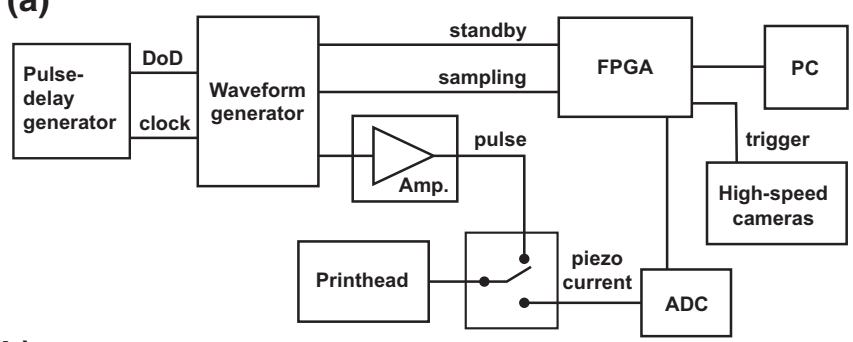

(b)

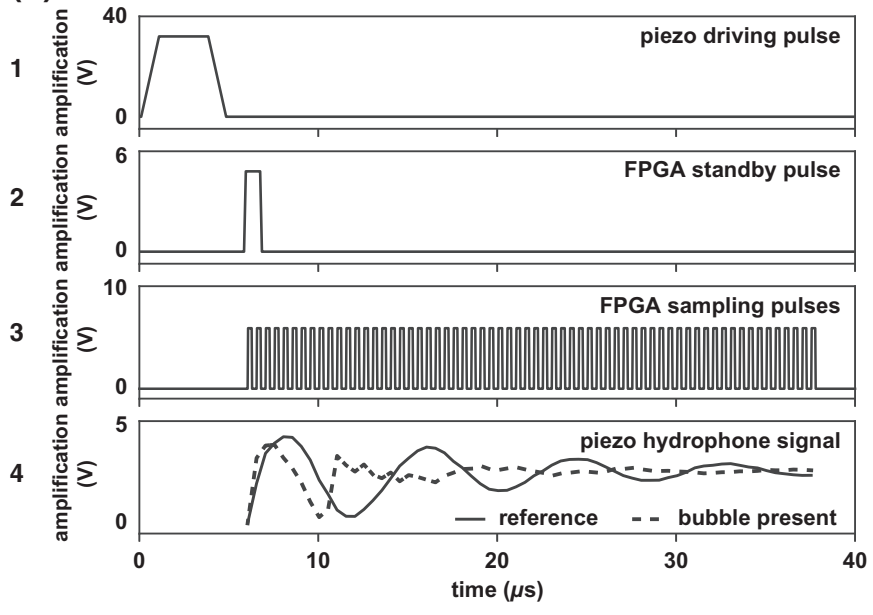

FIG. 10. (a) The control system that actuates the printhead and monitors the ink-channel acoustics to trigger the imaging system at a bubble-entrainment event. (b) (1-3) Waveforms created by the waveform generator; (4) two piezocurrent signals measured by the FPGA, i.e., a reference signal and a signal in the event that a bubble has been entrained.

is used to communicate with the FPGA via the personal computer (PC).

\section{APPENDIX D: PROBABILITY DISTRIBUTION OF DIRT-PARTICLE POSITION}

To measure the particle positions from the recorded images, the images are first divided by a background image in which no dirt particles are present. Then the images are binarized and noise is removed by excluding regions smaller than 5 pixels $(3 \mu \mathrm{m})$ for further analysis. It is verified that the typical dirt-particle size is larger than $5 \mu \mathrm{m}$. The locations of the detected particles are stored in a matrix. Finally, the numbers of particles detected in grid cells of $3 \times 3 \mu \mathrm{m}^{2}$ are measured from this position matrix to find the probability of the dirt-particle positions in the ink channel.

\section{APPENDIX E: PARTICLE-TRACKING VELOCIMETRY}

To include the $\mathrm{SiO}$ particles in the hot-melt ink, the ink is first melted on a hot plate at $130^{\circ} \mathrm{C}$. Then, the $\mathrm{SiO}$ particles are added and thoroughly dispersed using a tip sonicator (Branson Sonifier). $\mathrm{SiO}$ particles are chosen since they do not melt at the operating temperature of the printhead, of $130^{\circ} \mathrm{C}$.

For the measurement of the velocity field from the recordings, first, each image is divided by a background image of the ink channel without particles. The resulting image is binarized using an intensity threshold. For each high-speed video frame, the detected particle positions are stored in a matrix. The resulting position matrix is processed using a tracking algorithm [56] to track individual particles over the frames. The time-averaged velocity field in the ink channel is obtained by averaging the calculated particle velocity over a grid of $10 \times 10$ pixels $\left(6 \times 6 \mu \mathrm{m}^{2}\right)$.

\section{APPENDIX F: NOZZLE-FAILURE STATISTICS}

To measure the number of successfully jetted droplets before nozzle failure, first, a high-speed recording at $200 \mathrm{frames} / \mathrm{s}$ is started (SA-X2 camera, spatial resolution $0.4 \mu \mathrm{m} /$ pixel). Then, the jetting process and the acoustic triggering system are started. Once the correlation coefficient of the acoustic signal drops below 0.8 for a duration of ten actuation cycles, the camera is triggered to stop recording. Note that only the cases where the nozzle completely fails are considered, i.e., the cases for which the bubbles are jetted outward within ten actuation cycles are not considered. The number of successfully jetted droplets is then calculated from the time between the frame number at which the channel is first actuated until the frame number at which a nucleated bubble is visible for the first time and from the DOD frequency. Given the 20-kHz DOD frequency and the 200 -frames/s recording rate, the error in the number of jetted droplets is 100 .

[1] H. Wijshoff, The dynamics of the piezo inkjet printhead operation, Phys. Rep. 491, 77 (2010).

[2] S. D. Hoath, Fundamentals of Inkjet Printing: The Science of Inkjet and Droplets (Wiley-VCH Verlag GmbH \& Co. KGaA, Weinheim, Germany, 2015).

[3] N.-T. Nguyen and S. T. Wereley, Fundamentals and Applications of Microfluidics (Artech House, Inc., Norwood, MA, 2002).

[4] S. Majee, M. Song, S.-L. Zhang, and Z.-B. Zhang, Scalable inkjet printing of shear-exfoliated graphene transparent conductive films, Carbon 102, 51 (2016).

[5] S. Majee, C. Liu, B. Wu, S.-L. Zhang, and Z.-B. Zhang, Ink-jet printed highly conductive pristine graphene patterns achieved with water-based ink and aqueous doping processing, Carbon 114, 77 (2017).

[6] S. K. Eshkalak, A. Cinnappan, W. A. D. M. Jayathilaka, M. Khatibzadeh, E. Kowsari, and S. Ramakrishna, A review on inkjet printing of CNT composites for smart applications, Appl. Mater. Today 9, 372 (2017).

[7] M. Vilardell, X. Granados, S. Ricart, I. Van Driessche, A. Palau, T. Puig, and X. Obradors, Flexible manufacturing 
of functional ceramic coatings by inkjet printing, Thin Solid Films 548, 489 (2013).

[8] A. Moya, G. Gabriel, R. Villa, and F. Javier del Campo, Inkjet-printed electrochemical sensors, Curr. Opin. Electrochem. 3, 29 (2017).

[9] T. M. Eggenhuisen, Y. Galagan, E. W. C. Coenen, W. P. Voorthuijzen, M. W. L Slaats, S. A. Kommeren, S. Shanmuganam, M. J. J. Coenen, R. Andriessen, and W. A. Groen, Digital fabrication of organic solar cells by inkjet printing using non-halogenated solvents, Sol. Energy Mater. Sol. Cells 134, 364 (2015).

[10] S. G. Hashmi, M. Ozkan, J. Halme, K. D. Misic, S. M. Zakeeruddin, J. Paltakari, M. Grätzel, and P. D. Lund, High performance dye-sensitized solar cells with inkjet printed ionic liquid electrolyte, Nano Energy 17, 206 (2015).

[11] T. Shimoda, K. Morii, S. Seki, and H. Kiguchi, Inkjet printing of light-emitting polymer displays, Inkjet Printing Funct. Mater. 28, 821 (2003).

[12] C. Jiang, L. Mu, J. Zou, Z. He, Z. Zhong, L. Wang, M. Xu, J. Wang, J. Peng, and Y. Cao, Full-color quantum dots active matrix display fabricated by ink-jet printing, Sci. China Chem. 60, 1349 (2017).

[13] R. Daly, T. S. Harrington, G. D. Martin, and I. M. Hutchings, Inkjet printing for pharmaceutics - a review of research and manufacturing, Int. J. Pharm. 494, 554 (2015).

[14] A. Simaite, F. Mesnilgrente, B. Tondu, P. Souères, and C. Bergaud, Towards inkjet printable conducting polymer artifical muscles, Sens. Actuators B: Chem. 229, 425 (2016).

[15] S. Hewes, A. D. Wong, and P. C. Searson, Bioprinting microvessels using and inkjet printer, Bioprinting 7, 14 (2017).

[16] M. Nakamura, A. Kobayashi, F. Takagi, A. Watanabe, Y. Hiruma, K. Ohuchi, Y. Iwasaki, M. Horie, I. Morita, and S. Takatani, Biocompatible inkjet printing technique for designed seeding of individual living cells, Tissue Eng. 11, 1658 (2005).

[17] G. Villar, A. D. Graham, and H. Bayley, A tissue-like printed material, Science 340, 48 (2013).

[18] B. Derby, Additive manufacture of ceramic components by inkjet printing, Engineering 1, 113 (2015).

[19] A. A. Khalate, X. Bombois, G. Scorletti, R. Babuska, S. Koekebakker, and W. de Zeeuw, A waveform design method for a piezo inkjet printhead based on robust feedforward control, J. Microelectromech. Syst. 21, 1365 (2012).

[20] C. Menzel, A. Bibl, and P. Hoisington, MEMS solutions for precision micro-fluidic dispensing application, Technical Report, Fujifilm Dimatix Inc. (2004).

[21] B.-H. Kim, T.-G. Kim, T.-K. Lee, S. Kim, S.-J. Shin, S.-J. Kim, and S.-J. Lee, Effects of trapped air bubbles on frequency responses of the piezo-driven inkjet printheads and visualization of the bubbles using synchrotron X-ray, Sens. Actuators A: Phys. 154, 132 (2009).

[22] A. van der Bos, T. Segers, R. Jeurissen, M. van den Berg, H. Reinten, H. Wijshoff, M. Versluis, and D. Lohse, Infrared imaging and acoustic sizing of a bubble inside a micro-electro-mechanical system piezo ink channel, J. Appl. Phys. 110, 034503 (2011).

[23] B.-H. Kim, H.-S. Lee, S.-W. Kim, P. Kang, and Y.-S. Park, Hydrodynamic responses of a piezoelectric driven MEMS inkjet print-head, Sens. Actuators A: Phys. 210, 131 (2014).
[24] J. F. Dijksman, Hydro-acoustics of piezoelectrically driven ink-jet print heads, Flow, Turbulence Combust. 61, 211 (1998).

[25] J. de Jong, G. de Bruin, H. Reinten, M. van den Berg, H. Wijshoff, M. Versluis, and D. Lohse, Air entrapment in piezo-driven inkjet printheads, J. Acoustical Soc. Am. 120, 1257 (2006).

[26] J. de Jong, R. Jeurissen, H. Borel, M. van den Berg, H. Wijshoff, H. Reinten, M. Versluis, A. Prosperetti, and D. Lohse, Entrapped air bubbles in piezo-driven inkjet printing: Their effect on the droplet velocity, Phys. Fluids 18, 121511 (2006).

[27] R. Jeurissen, J. de Jong, H. Reinten, M. van den Berg, H. Wijshoff, M. Versluis, and D. Lohse, Effect of an entrained air bubble on the acoustics of an ink channel, J. Acoustical Soc. Am. 123, 2496 (2008).

[28] R. Jeurissen, A. van der Bos, H. Reinten, M. van den Berg, H. Wijshoff, J. de Jong, M. Versluis, and D. Lohse, Acoustic measurement of bubble size in an inkjet printhead, J. Acoustical Soc. Am. 126, 2184 (2009).

[29] S. J. Lee, D. H. Kwon, and Y. S. Choi, Dynamics of entrained air bubbles inside a piezodriven inkjet printhead, Appl. Phys. Lett. 95, 221902 (2009).

[30] R. Jeurissen, H. Wijshoff, M. van den Berg, H. Reinten, and D. Lohse, Regimes of bubble volume oscillations in a pipe, J. Acoustical Soc. Am. 130, 3220 (2011).

[31] L. A. Crum, Rectified diffusion, Ultrasonics 22, 215 (1984).

[32] T. G. Leighton, The Acoustic Bubble (Academic Press, London, 1994).

[33] C. E. Brennen, Cavitation and Bubble Dynamics (Oxford University Press, New York, 1995).

[34] M. P. Brenner, S. Hilgenfeldt, and D. Lohse, Single-bubble sonoluminescence, Rev. Mod. Phys. 74, 425 (2002).

[35] L. Rayleigh, Investigation of the character of the equilibrium of an incompressible heavy fluid of variable density, Proc. London Math. Soc. 14, 170 (1883).

[36] G. Taylor, The instability of liquid surfaces when accelerated in a direction perpendicular to their planes. I, Proc. R. Soc. London A 201, 192 (1950).

[37] M. Faraday, On a peculiar class of acoustical figures; and on certain forms assumed by groups of particles upon vibrating elastic surfaces, Philos. Trans. R. Soc. London 121, 299 (1831).

[38] J. R. Castrejón-Pita, G. D. Martin, and I. M. Hutchings, Experimental study of the influence of nozzle defects on drop-on-demand ink jets, J. Imaging Sci. Technol. 55, 40305 (2011).

[39] A. Fraters, T. Segers, M. van den Berg, H. Reinten, H. Wijshoff, D. Lohse, and M Versluis, Shortwave infrared imaging setup to study entrained air bubble dynamics in a MEMS-based piezo-acoustic inkjet printhead, Exp. Fluids 60, 123 (2019).

[40] T. Segers and M. Versluis, Acoustic bubble sorting for ultrasound contrast agent enrichment, Lab Chip 14, 1705 (2014).

[41] M. Overvelde, H. J. Vos, N. de Jong, and M. Versluis, in Ultrasound Contrast Agents: Targeting and Processing Methods for Theranostics (Springer Milan, Milan, 2010).

[42] See the Supplemental Material at http://link.aps.org/supple mental/10.1103/PhysRevApplied.12.064019 for the videos of Figs. 3, 4, 6, 7, and 8. 
[43] J. Lighthill, Acoustic streaming, J. Sound Vib. 61, 391 (1978).

[44] P. Marmottant and S. Hilgenfeldt, A bubble-driven microfluidic transport element for bioengineering, Proc. Natl. Acad. Sci. USA 101, 9523 (2004).

[45] R. Bolanos-Jimenéz, M. Rossi, D. Fernandez Rivas, C. J. Kähler, and A. Marin, Streaming flow by oscillating bubbles: Quantitative diagnostics via particle tracking velocimetry, J. Fluid Mech. 820, 529 (2017).

[46] G. Lajoinie, Y. Luan, E. Gelderblom, B. Dollet, F. Mastik, H. Dewitte, I. Lentacker, N. de Jong, and M. Versluis, Non-spherical oscillations drive the ultrasound-mediated release from targeted microbubbles, Commun. Phys. 1, 22 (2018).

[47] H. J. Herrmann, J. S. Andrade, Jr., A. D. Araújo, and M. P. Almeida, Particles in fluids, Eur. Phys. J. Spec. Top. 143, 181 (2007).

[48] B. R. Munson, D. F. Young, T. H. Okiishi, and W. W. Huebsch, Fundamentals of Fluid Mechanics (Wiley, Jefferson City, 2009), 6th ed.
[49] A. J. Mach, J. H. Kim, A. Arshi, S. C. Hur, and D. Di Carlo, Automated cellular sample preparation using a centrifuge-on-a-chip, Lab Chip 11, 2827 (2011).

[50] T. Karino and H. L. Goldsmith, Flow behaviour of blood cells and rigid spheres in an annular vortex, Philos. Trans. R. Soc. London B: Biol. Sci. 279, 413 (1977).

[51] J. Zhang, S. Yan, D. Yuan, G. Alici, N.-T. Nguyen, M. E. Warkiani, and W. Li, Fundamentals and applications of inertial microfluidics: A review, Lab Chip 16, 10 (2016).

[52] J. Rensen, D. Bosman, J. Magnaudet, C.-D. Ohl, A. Prosperetti, R. Tögel, M. Versluis, and D. Lohse, Spiraling Bubbles: How Acoustic and Hydrodynamic Forces Compete, Phys. Rev. Lett. 86, 4819 (2001).

[53] D. Lohse, Bubble puzzles: From fundamentals to applications, Phys. Rev. Fluids 3, 110504 (2018).

[54] L. A. Crum, Bjerknes forces on bubbles in a stationary sound field, J. Acoustical Soc. Am. 57, 1363 (1975).

[55] B. Illowsky and S. Dean, Introductory Statistics (OpenStax CNX, Houston, Texas, 2018).

[56] D. Blair and E. Dufresne, The MATLAB particle tracking code repository, http://site.physics.georgetown.edu/matlab/. 